\title{
Eine scheinbare Abschwächung der Lokalitätsbedingung
}

\author{
K. Pohlmeyer
}

II. Institut für Theoretische Physik der Universität Hamburg

Eingegangen am 21. Juli 1967

\begin{abstract}
For a hermitean, scalar, tempered field $A(x)$ the locality axiom can be replaced by the following condition: For any two natural numbers $n$ and $j$ with $1 \leqq j<n$ and for any configuration $X(n, j): X_{1}, \ldots, X_{j-1}, X_{j}, X_{j+1}, X_{j+2}, \ldots, X_{n}$ that is totally space-like in both orders: $1, \ldots, j-1, j, j+1, j+2, \ldots, n$ and $1, \ldots, j-1, j+1, j, j+2, \ldots, n$ there exist constants $\alpha(n, j)>2, C(X(n, j))>0$, $h(X(n, j))>0$ such that with $x_{k}=X_{k} \sqrt{-x^{2}}$ :

$\left|\left\langle A\left(x_{1}\right) \ldots A\left(x_{j-1}\right)\left[A\left(x_{j}\right), A\left(x_{j+1}\right)\right] A\left(x_{j+2}\right) \ldots A\left(x_{n}\right)\right\rangle\right|<$

for $-x^{2}>1$.$$
<C(X(n, j)) \exp \left\{-h(X(n, j)) \sqrt{-x^{2}}{ }^{\alpha}(n, j)\right\}
$$

\section{Einleitung}

Es ist bekannt [1], daß ein hermitesches, skalares Feld $A(x)$, das die Wightman Axiome erfüllt mit evtl. Ausnahme des Lokalitätsaxioms, strikt lokal ist, falls gilt:

$$
[A(x), A(y)]=0,
$$

wenn $x$ und $y$ irgendzwei offene, raumartig getrennte Mengen durchlaufen.

In der hier vorliegenden Arbeit wollen wir die Frage diskutieren, ob es für echt nichtlokale Theorien eine Schranke für den Abfall der Beiträge zum Kommutator aus dem akausalen Bereich für große raumartige Abstände $r$ gibt (d. h. aus dem Bereich außerhalb des Lichtkegels). Wie zunächst an den Beispielen der Drei- und Vierpunktfunktion ${ }^{1}$, dann aber in voller Allgemeinheit gezeigt werden soll, gibt es solche Schranken, so da $\beta$ das Feld $A(x)$ strikt lokal ist, falls - grob gesprochen - die Beiträge zum Kommutator aus dem akausalen Bereich wie

mit $\alpha>2$ abfallen.

$$
\text { const. } \exp \left(- \text { const }^{\prime} \cdot r^{\alpha}\right)
$$

1 Bekanntlich gibt ja die Lokalität keine Einschränkung für die Zweipunktfunktion. 


\section{Dreipunktfunktion}

Wir gehen aus von reellen Punkten $x_{1}, x_{2}, x_{3}$, die in dem Durchschnitt der "extended tubes" der Dreipunktfunktionen $\left\langle A\left(x_{1}\right) A\left(x_{2}\right) A\left(x_{3}\right)\right\rangle$ und $\left\langle A\left(x_{2}\right) A\left(x_{1}\right) A\left(x_{3}\right)\right\rangle$ liegen. Wie R. Jost gezeigt hat [2], sind das gerade die in der Reihenfolge 1,2,3 bzw. 2, 1, 3 total raumartigen Punkte. Diese Punkte erfüllen also die beiden Ungleichungen

$$
\left(\mu_{1}\left(x_{1}-x_{2}\right)+\mu_{2}\left(x_{2}-x_{3}\right)\right)^{2}<0 \text { für alle } \mu_{i} \geqq 0, \Sigma \mu_{i}>0
$$

und

$$
\left(\lambda_{1}\left(x_{2}-x_{1}\right)+\lambda_{2}\left(x_{1}-x_{3}\right)\right)^{2}<0 \text { für alle } \lambda_{j} \geqq 0, \Sigma \lambda_{j}>0 .
$$

Es soll nun zunächst dargelegt werden, daß diese Punkte ohne Beschränkung der Allgemeinheit als gleichzeitig angenommen werden dürfen. Andernfalls können wir nämlich durch Translationen und Lorentzdrehungen erreichen, daß $x_{1}, x_{2}, x_{3}$ folgende Gestalt haben:

$$
x_{1}=\left(\begin{array}{l}
0 \\
0 \\
0 \\
0
\end{array}\right), \quad x_{2}=\left(\begin{array}{l}
0 \\
x_{2}{ }^{1} \\
0 \\
0
\end{array}\right), \quad x_{3}=\left(\begin{array}{c}
x_{3}{ }^{0} \\
x_{3}{ }^{1} \\
x_{3}{ }^{2} \\
0
\end{array}\right) .
$$

Wenn wir nun zeigen können, daß $\left|x_{3}^{2}\right|>\left|x_{3}^{0}\right|$, brauchen wir nur eine Lorentztransformation in der 0,2-Ebene anzuwenden, um die angestrebte Form zu erreichen. Die Ungleichung $\left|x_{3}^{2}\right|>\left|x_{3}^{0}\right|$ folgt aber aus der totalen Raumartigkeit in den beiden Reihenfolgen 1,2,3 bzw. 2, 1, 3, denn sei $\left(x_{2}^{1} \neq 0\right.$, wegen $\left.\left(1 .\left(0-x_{2}\right)+0 .\left(x_{2}-x_{3}\right)\right)^{2}<0\right)$

Fall 1: $\frac{x_{3}{ }^{1}}{x_{2}{ }^{1}} \leqq 0$.

Dann wähle man

und im

$$
\mu_{1}=1-\frac{x_{3}{ }^{1}}{x_{2}{ }^{1}} \geqq 1 \quad \mu_{2}=1
$$

Fall 2: $\frac{x_{3}{ }^{1}}{x_{2}{ }^{1}}>0$

wähle man

$$
\lambda_{1}=\frac{x_{3}{ }^{1}}{x_{2}{ }^{1}} \quad \lambda_{2}=1
$$

wodurch aus den Ungleichungen (1) bzw. (2) wird: $\left(\begin{array}{c}-x_{3}{ }^{0} \\ 0 \\ -x_{3}{ }^{2} \\ 0\end{array}\right)^{2}<0$, d. h. $\left|x_{3}^{2}\right|>\left|x_{3}^{0}\right| \cdot$

Damit sehen wir, daß die Form $x_{1}^{0}=x_{2}^{0}=x_{3}^{0}=0$ keine Einschränkung der Allgemeinheit bedeutet.

Totale Raumartigkeit (in beiden Reihenfolgen) für 3 gleichzeitige Punkte $x_{1}, x_{2}, x_{3}$ ist aber äquivalent mit der Aussage, daß die Dreiervektoren $\vec{x}_{1}-\vec{x}_{2}, \vec{x}_{2}-\vec{x}_{3}$ linear unabhängig sind. Diese Vektoren spannen nun eine Ebene auf, in die wir die $x^{1}$ - und $x^{2}$-Achsen unseres Koordi6 Commun. math. Phys., Vol. 7 
natensystems legen wollen, so daß die $x^{1}$-Achse auf den Vektor $\vec{x}_{2}-\vec{x}_{1}$ fällt und $x_{3}^{2}>0$. Insgesamt also bedeutet für die betrachteten Punkte die Form

$$
x_{1}=\left(\begin{array}{l}
0 \\
0 \\
0 \\
0
\end{array}\right), \quad x_{2}=\left(\begin{array}{l}
0 \\
x_{2}{ }^{1} \\
0 \\
0
\end{array}\right), \quad x_{3}=\left(\begin{array}{l}
0 \\
x_{3}{ }^{1} \\
x_{3}{ }^{2} \\
0
\end{array}\right), \quad x_{2}^{1}>0, x_{3}^{2}>0
$$

keine Beschränkung der Allgemeinheit.

Wir wollen nun die Seitenlängen des von den 3 Punkten gebildeten Dreiecks in folgender Weise von dem Differenzvektor $x=x_{1}-x_{2}$ abhängen lassen :

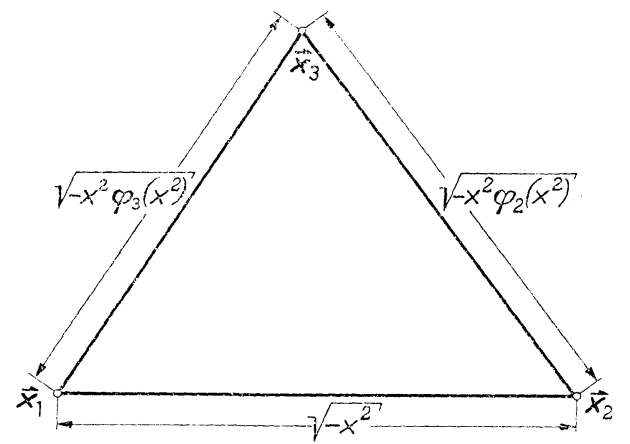

Fig. 1

wobei an die Funktionen $\varphi_{2}(z)$ und $\varphi_{3}(z) z=x+i y$ folgende Forderungen gestellt werden:

$\varphi_{2}(z)$ und $\varphi_{3}(z)$ sollen in dem Winkelbereich $\Theta_{1}<\Theta=\arg z<2 \pi-\Theta_{1}$ $=\Theta_{3}$ analytische Funktionen sein, für welche für reelle $z<0$ gilt:

$$
\varphi_{2}(z), \varphi_{3}(z) \text { reell, }>0
$$

$1-2 \sqrt{\varphi_{2}(z)}+\varphi_{2}(z)<\varphi_{3}(z)<1+2 \sqrt{\varphi_{2}(z)}+\varphi_{2}(z) \quad$ (Dreiecksungleichung). Weiterhin sollen $\varphi_{2}(z)=\overline{p_{2}(\bar{z})}$ und $\varphi_{3}(z)=\overline{\varphi_{3}(\bar{z})}$ in $\Theta_{1}<\Theta<2 \pi-\Theta_{1}$ den folgenden Bedingungen genügen:

(a)

$$
\begin{array}{lll} 
& z \varphi_{2}(z) \neq z(1-k)+z \varphi_{3}(z)\left(1-\frac{1}{k}\right) & 0<k<\infty \\
\text { (a) } & z \varphi_{3}(z) \neq z\left(1-k^{\prime}\right)+z \varphi_{2}(z)\left(1-\frac{1}{k^{\prime}}\right) & 0<k^{\prime}<\infty \\
& z \varphi_{2}(z) \neq \text { reell, }>0 \\
\text { (b) } & z \varphi_{3}(z) \neq \text { reell, }>0 \\
& z \varphi_{3}(z) \neq z+z \varphi_{2}(z)+r+\frac{z^{2} \varphi_{2}(z)}{r} \quad 0<r<\infty \\
\text { (c) } & z \varphi_{2}(z) \neq z+z \varphi_{3}(z)+r^{\prime}+\frac{z^{2} \varphi_{3}(z)}{r^{\prime}} & 0<r^{\prime}<\infty .
\end{array}
$$


Den mit Analytizitätsbetrachtungen von Wightmanfunktionen vertrauten Leser werden diese Bedingungen an die von G. KäLLÉN und A. S. Wightman [3] gefundenen Berandungen der "extended tubes" der Dreipunktfunktionen $W(1,2,3)=\left\langle A\left(x_{1}\right) A\left(x_{2}\right) A\left(x_{3}\right)\right\rangle$ bzw. $W(2,1,3)$ $=\left\langle A\left(x_{2}\right) A\left(x_{1}\right) A\left(x_{3}\right)\right\rangle$ erinnern. Man setze nur: $z_{1}=z, z_{2}=z \varphi_{2}(z), z_{3}$ $=z \varphi_{3}(z)$. Tatsächlich beschäftigen wir uns auch im Augenblick mit nichts anderem, als Suchlinien für die Funktion $W(1,2,3)-W(2,1,3)$ zu konstruieren, deren Analytizitätsbereich den Durchschnitt der "extended tubes" von $W(1,2,3)$ und $W(2,1,3)$ umfaßt.

Für die folgenden Betrachtungen von Interesse ist die Frage nach dem größten Winkelbereich $\Theta_{1}<\Theta<2 \pi-\Theta_{1}=\Theta_{3}$, bzw. nach dem kleinsten Winkel $\Theta_{1}$, für den noch solche Funktionen $\varphi_{2}$ und $\varphi_{3}$ existieren. Diese Frage wird hier nicht in voller Allgemeinheit beantwortet werden. Unter den zusätzlichen Voraussetzungen

$\frac{\varphi_{3}(z)-\varphi_{2}(z)}{\sqrt{\varphi_{2}(z)}} \rightarrow$ const. bzw. $\frac{\varphi_{3}(z)-\varphi_{2}(z)}{\sqrt{\varphi_{2}(z)}} \rightarrow$ const.' $^{\prime}$

und

für $z \rightarrow-\infty$ bzw. $z=|z| e^{i\left(\Theta_{1}+0\right)},|z| \rightarrow \infty$

$$
\varphi_{2}(z) \rightarrow\{\infty\} \text { oder } \varphi_{2}(z) \rightarrow \text { const." für } \Theta_{1}<\Theta<2 \pi-\Theta_{1},
$$

stellt sich heraus: $\Theta_{1} \geqq \frac{\pi}{2}$. Das liegt u. a. daran, daß sich auf der einen Seite die Phasen von $\varphi_{2}$ und $\varphi_{3}$ in Abhängigkeit von arg z nicht zu schnell ändern dürfen, andererseits aber die Dreiecksungleichungen gelten sollen und Herglotzfunktionen höchstens mit $|z|$ ansteigen.

Es ist nicht gänzlich ausgeschlossen, daß es Suchlinien gibt, die nicht diese zusätzlichen Voraussetzungen erfüllen und zu denen ein größerer Winkelbereich bzw. ein kleineres $\Theta_{1}$ gehört, denn die Winkelöffnung der Menge

$P=\left\{z_{1} \mid \exists z_{2}, z_{3}\right.$ s.d. $\left(z_{1}, z_{2}, z_{3}\right) \in$ Regularitätsbereich

beträgt $2 \pi-0$.

$$
\text { von } W(1,2,3)-W(2,1,3)\}
$$

In Anbetracht der Grenze $\Theta_{1}=\frac{\pi}{2}$ für Suchlinien aus der oben erklärten Klasse können wir uns auf die einfachsten Suchlinien zurückziehen, nämlich

$$
\begin{gathered}
\varphi_{2}(z)=a_{2}=\text { const., } \varphi_{3}(z)=a_{3}=\text { const. } \\
a_{2}, a_{3}>0: 1-2 \sqrt{a_{2}}+a_{2}<a_{3}<1+2 \sqrt{a_{2}}+a_{2}
\end{gathered}
$$


d. h. wir betrachten die folgenden Vektortripel

$$
x_{1}=\left(\begin{array}{l}
0 \\
0 \\
0 \\
0
\end{array}\right), \quad x_{2}=\left(\begin{array}{c}
0 \\
\sqrt{-z} \\
0 \\
0
\end{array}\right), \quad x_{3}=\left(\begin{array}{c}
\frac{a_{3}-a_{2}+1}{2} \sqrt{-z} \\
\sqrt{a_{3}-\left[\frac{a_{3}-a_{2}+1}{2}\right]^{2}} \sqrt{-z} \\
0
\end{array}\right) .
$$

Der zu dieser Situation gehörige Grenzwinkel $\Theta_{1}(a)$ ist

$$
\begin{aligned}
& \pi-\operatorname{arctg} \sqrt{\frac{4 a_{3}}{\left(a_{3}-a_{2}+1\right)^{2}}-1} \quad \text { falls } a_{2}-a_{3}-1<0, a_{3}-a_{2}-1>0 \\
& \pi-\operatorname{arctg} \sqrt{\frac{4 a_{2}}{\left(a_{2}-a_{3}+1\right)^{2}}-1} \quad \text { falls } a_{2}-a_{3}-1>0, a_{3}-a_{2}-1<0 \\
& \pi-\operatorname{Min}\left\{\operatorname{arctg} \sqrt{\frac{4 a_{3}}{\left(a_{3}-a_{2}+1\right)^{2}}-1}, \operatorname{arctg} \sqrt{\frac{4 a_{2}}{\left(a_{2}-a_{3}+1\right)^{2}}-1}\right\} \\
& \quad \text { falls } a_{2}-a_{3}-1<0, a_{3}-a_{2}-1<0 .
\end{aligned}
$$

Wir sind nun in der Lage, unser erstes Theorem zu formulieren und zu beweisen:

Theorem 1. Sei $A(x)$ ein hermitesches, skalares Feld, das die Wightman Axiome erfüllt mit evtl. Ausnahme des Lokalitätsaxioms, welches wir durch die folgende Voraussetzung ersetzen wollen:

$Z u$ jedem Punkt $(a)=\left(a_{2}, a_{3}\right) \in M=\left\{\left(a_{2}, a_{3}\right) \mid a_{2}, a_{3}>0,1-2 \sqrt{a_{2}}+\right.$ $\left.+a_{2}<a_{3}<1+2 \sqrt{a_{2}}+a_{2}\right\} \subset R^{2}$ gibt es Konstanten $C(a), h(a)>0$, so da $\beta$ (V 1) $\left|\left\langle\left[A\left(x_{1}\right) A\left(x_{2}\right)\right] A\left(x_{3}\right)\right\rangle\right|\left\langle C(a) \exp \left\{-h(a) \sqrt{-z^{\alpha}}\right\}\right.$

$$
\text { mit } x_{1}=\left(\begin{array}{l}
0 \\
0 \\
0 \\
0
\end{array}\right), \quad x_{2}=\left(\begin{array}{c}
0 \\
\sqrt{-z} \\
0 \\
0
\end{array}\right), \quad x_{3}=\left(\begin{array}{c}
\frac{a_{3}-a_{2}+1}{2} \sqrt{-z} \\
\sqrt{\frac{a_{3}-\left[\frac{a_{3}-a_{2}+1}{2}\right]^{2}}{2} \sqrt{-z}}
\end{array}\right)
$$

für reelles $-z>1$.

Dann folgt für $\alpha>2$ :

$$
\begin{array}{ll}
\left\langle\left[A\left(x_{1}\right), A\left(x_{2}\right)\right] A\left(x_{3}\right)\right\rangle=0 & \text { für alle reellen Punkte } x_{1}, x_{2}, x_{3} \\
& \text { mit }\left(x_{1}-x_{2}\right)^{2}<0 .
\end{array}
$$

Beweis. Für $\Theta_{1}(a)<\Theta<2 \pi-\Theta_{1}(a)$ mit

$$
\Theta_{1}(a)=\left\{\begin{array}{l}
\pi-\operatorname{arctg} \sqrt{\frac{4 a_{3}}{\left(a_{3}-a_{2}+1\right)^{2}}-1} \text { falls } a_{2}-a_{3}-1<0, a_{3}-a_{2}-1>0 \\
\pi-\operatorname{arctg} \sqrt{\frac{4 a_{2}}{\left(a_{2}-a_{3}+1\right)^{2}}-1} \text { falls } a_{2}-a_{3}-1>0, a_{3}-a_{2}-1<0 \\
\pi-\operatorname{Min}\left\{\operatorname{arctg} \sqrt{\frac{4 a_{3}}{\left(a_{3}-a_{2}+1\right)^{2}}-1}, \operatorname{arctg} \sqrt{\frac{4 a_{2}}{\left(a_{2}-a_{3}+1\right)^{2}}-1}\right\} \\
\text { falls } a_{2}-a_{3}-1<0, a_{3}-a_{2}-1<0
\end{array}\right.
$$


liegen die Vektoren

$$
x_{1}=\left(\begin{array}{l}
0 \\
0 \\
0 \\
0
\end{array}\right), \quad x_{2}=\left(\begin{array}{c}
0 \\
\sqrt{-z} \\
0 \\
0
\end{array}\right), \quad x_{3}=\left(\begin{array}{c}
\frac{a_{3}-a_{2}+1}{2} \sqrt{-z} \\
\sqrt{\frac{a_{3}-\left[\frac{a_{3}-a_{2}+1}{2}\right]}{2}} \sqrt{-z}
\end{array}\right)
$$

im Regularitätsgebiet von $\{W(1,2,3)-W(2,1,3)\}(z)$. Für $\alpha>2$ können wir eine offene Menge $O$ finden: $O \subset M$, so daß $\Theta_{1}(a)<\pi-\frac{\pi}{\alpha}$ für alle $(a) \in O$. Führen wir nun statt der Variablen $z$ die Variable $\zeta=z^{\alpha / 2}$ ein, so ist

$$
W_{a_{2}, a_{3}}^{C}(\zeta)=\{W(1,2,3)-W(2,1,3)\}_{a_{2}, a_{3}}(\zeta)
$$

in einem Winkelbereich mit der Öffnung $>\pi$ regulär. Wegen unserer Voraussetzung ( $\mathrm{V}$ l) und der Temperiertheitsannahme für das Feld $A(x)$ ergibt sich somit folgende Situation für $\left(a_{2}, a_{3}\right) \in O$ :

$W_{a_{2}, a_{3}}^{C}(\zeta)$ regulär in $\Theta_{1} \leqq \Theta \leqq \Theta_{1}+\pi+\varepsilon=\Theta_{3}, \varepsilon>0$ und dort polynomial beschränkt, also erst recht von exponentiellem Typ.

Auf den Strahlen $\Theta=\Theta_{1}$ und $\Theta=\Theta_{3}$ gilt:

$$
\limsup _{|\zeta| \rightarrow \infty}\left\{|\zeta|^{-1} \log \left|W_{a_{2}, a_{3}}^{C}\left(|\zeta| e^{i \Theta}\right)\right|\right\}=0,
$$

aber auf dem Strahl $\Theta=\Theta_{2}=\frac{\pi \alpha}{2}, \Theta_{1}<\Theta_{2}<\Theta_{3}$ gilt:

$$
\limsup _{|\xi| \rightarrow \infty}\left\{|\xi|^{-1} \log \left|W_{a_{2}, a_{3}}^{C}\left(|\xi| e^{i \Theta}\right)\right|\right\}<0 .
$$

Nach einem Theorem über Funktionen von exponentiellem Typ besteht die Klasse solcher Funktionen nur aus der 0 [4]:

$$
W\left(z_{1}, a_{2} z_{1}, a_{3} z_{1}\right)-W\left(z_{1}, a_{3} z_{1}, a_{2} z_{1}\right)=0 \text { für alle }\left(a_{2}, a_{3}\right) \in O .
$$

Die Punkte $z_{1}, a_{2} z_{1}, a_{3} z_{1}$ und $z_{1}, a_{3} z_{1}, a_{2} z_{1}$ liegen nun für $\Theta_{1}(a)<\Theta$ $=\arg z_{1}<2 \pi-\Theta_{1}(a)$ und $\left(a_{2}, a_{3}\right) \in O$ im (offenen) Kern des Bildes des Durchschnitts der "extended tubes": $I\left(\mathscr{T}_{1,2,3}^{\prime} \cap \mathscr{T}_{2,1,3}^{\prime}\right)$, wobei $I$ die Abbildung auf die Invarianten ist.

Die Transformation: $a_{2}=\frac{z_{2}}{z_{1}}, a_{3}=\frac{z_{3}}{z_{1}}$ bzw. $a_{3}=\frac{z_{2}}{z_{1}}, a_{2}=\frac{z_{3}}{z_{1}}$ ist regulär für $z_{1} \neq 0$. Daraus folgt:

$$
W\left(z_{1}, z_{2}, z_{3}\right)-W\left(z_{1}, z_{3}, z_{2}\right) \equiv 0
$$

in $I\left(\mathscr{T}_{1,2,3}^{\prime} \cap \mathscr{T}_{2,1,3}^{\prime}\right)$.

Von diesem Punkt an braucht man nur die Argumente zum Beweis des Theorems 4-1 von [3] zu wiederholen, um zu dem Schluß zu gelangen:

a) $\left\langle\left[A\left(x_{1}\right), A\left(x_{2}\right)\right] A\left(x_{3}\right)\right\rangle=0$ für alle reellen Punkte $x_{1}, x_{2}, x_{3}$ mit $\left(x_{1}-x_{2}\right)^{2}<0$. Bei der Behandlung von $\left\langle A\left(x_{1}\right)\left[A\left(x_{2}\right), A\left(x_{3}\right)\right]\right\rangle-$ wir 
machen die (V 1) entsprechende Voraussetzung - gehen wir analog vor und folgern:

b) $\left\langle A\left(x_{1}\right)\left[A\left(x_{2}\right), A\left(x_{3}\right)\right]\right\rangle=0$ für alle reellen Punkte $x_{1}, x_{2}, x_{3}$ mit $\left(x_{2}-x_{3}\right)^{2}<0$.

a) und b) bedeuten aber gerade strikte Lokalität für die Dreipunktfunktion.

Felder mit Akausalitäten von der Art (V 1) führen auf dieselben Eigenschaften für die Dreipunktfunktion wie strikt lokale Felder.

\section{Vierpunktfunktion}

Im Fall der Vierpunktfunktion sind wir nicht in der glücklichen Lage, die Charakterisierung der "extended tubes" in den Invarianten explizit angeben zu können (wohl aber kennen wir Teile von den Berandungen der "extended tubes" in den Invarianten: DANAD-Flächen). Wir werden daher in diesem Fall statt mit den Invarianten mit den Vierervektoren selbst arbeiten. Zum Nachweis dafür, daß gewisse Vektoren $(x+i y)_{l} l$ $=1,2,3,4$ in der "extended tube" zu der Reihenfolge 1, 2, 3, 4 liegen, müssen wir zeigen, daß es zu den Differenzvektoren $\zeta_{l}=(x+i y)_{l}-$ - $(x+i y)_{l+1} l=1,2,3 \mathrm{SL}(2, \mathrm{C})$-Matrizen $A$ und $B$ gibt, so daß

$$
\zeta_{l}^{\prime}=A \zeta_{l} B^{T} \quad \zeta \leftrightarrow \zeta=\left(\begin{array}{cc}
\zeta^{0}+\zeta^{3} & \zeta^{1}-i \zeta^{2} \\
\zeta^{1}+i \zeta^{2} & \zeta^{0}-\zeta^{3}
\end{array}\right)
$$

in der "primitive tube" (zu der Reihenfolge 1, 2, 3,4) liegt.

Da aber andererseits die "primitive tube" invariant ist unter reellen Lorentztransformationen ( $\zeta^{\prime}=A \zeta A^{*}, A \in S L(2, C)$ ), genügt es, die Existenz von $S L(2, C)$-Matrizen $C$ nachzuweisen mit der Eigenschaft

$$
\zeta_{l}^{\prime}=\zeta_{l} C \in \text { "primitive tube". }
$$

Als Beispiel geben wir die $S L(2, C)$-Matrizen $C_{1,2,3}$ und $C_{2,1,3}$ an, die die Punkte $\left(a_{2}=a_{3}=1\right)$

$$
\left(\begin{array}{l}
0 \\
0 \\
0 \\
0
\end{array}\right),\left(\begin{array}{c}
\frac{0}{\sqrt{-z}} \\
0 \\
0
\end{array}\right),\left(\begin{array}{c}
0 \\
\frac{1}{2} \sqrt{-z} \\
\frac{1}{2} \sqrt{3} \sqrt{-z} \\
0
\end{array}\right)
$$

für $\frac{2}{3} \pi<\Theta<\frac{4}{3} \pi$ in die "primitive tube" zu der Reihenfolge 1, 2, 3 bzw. 2, 1, 3 überführen:

$$
C_{1,2,3}=\left(\begin{array}{cc}
0 & \frac{\sqrt{3}}{2}-\frac{i}{2} \\
-\frac{\sqrt{3}}{2}-\frac{i}{2} & 0
\end{array}\right), \quad C_{2,1,3}=\left(\begin{array}{cc}
0 & \frac{\sqrt{3}}{2}+\frac{i}{2} \\
-\frac{\sqrt{3}}{2}+\frac{i}{2} & 0
\end{array}\right) .
$$


Wir wollen nun versuchen, die bei der Dreipunktfunktion herangezogene Beweismethode auf den Fall der Vierpunktfunktion zu übertragen. Die untere Grenze für die exponentielle Abnahmeordnung kann sich bei dem Übergang von der Drei- zur Vierpunktsfunktion natürlich nicht verbessern. Wir werden diejenigen Suchlinien verwenden, die sich schon bei der Dreipunktsfunktion bewährt haben. Die Frage, ob diese Suchlinien auch im Fall der Vierpunktfunktion in gewissem Sinn optimal sind, wird sich nachträglich von selbst beantworten. Dabei wollen wir uns zunächst darüber klar werden, daß die Gleichzeitigkeit der Ausgangssituation (die im Fall der Dreipunktfunktion keine Beschränkung der Allgemeinheit bedeutete), hier die Allgemeinheit einschränkt. Denn 4 Punkte $x_{1}, \ldots, x_{4}$

die die Bedingungen

$$
x_{i}=\left(\begin{array}{c}
x_{i}^{0} \\
x_{i}^{1} \\
x_{i}^{2} \\
x_{i}^{3}
\end{array}\right),
$$

$\left(\mu_{1}\left(x_{1}-x_{2}\right)+\mu_{2}\left(x_{2}-x_{3}\right)+\mu_{3}\left(x_{3}-x_{4}\right)\right)^{2}<0$ für alle $\mu_{i} \geqq 0, \Sigma \mu_{i}>0$ $\left(\lambda_{1}\left(x_{1}-x_{3}\right)+\lambda_{2}\left(x_{3}-x_{2}\right)+\lambda_{3}\left(x_{2}-x_{4}\right)\right)^{2}<0$ für alle $\lambda_{j} \geqq 0, \Sigma \lambda_{j}>0$ erfüllen, sind z. B.

$$
x_{1}=\left(\begin{array}{l}
0 \\
0 \\
0 \\
0
\end{array}\right), \quad x_{2}=\left(\begin{array}{l}
0 \\
1 \\
0 \\
0
\end{array}\right), \quad x_{3}=\left(\begin{array}{l}
0 \\
0 \\
1 \\
0
\end{array}\right), \quad x_{4}=\left(\begin{array}{c}
\frac{1}{2} \\
2 \\
0 \\
0
\end{array}\right) .
$$

Könnten nun diese Punkte durch (reelle oder komplexe) Lorentztransformationen gleichzeitig gemacht werden, so müßte gelten:

$$
\sqrt{-\left(x_{4}-x_{1}\right)^{2}} \leqq \sqrt{-\left(x_{4}-x_{2}\right)^{2}}+\sqrt{-\left(x_{2}-x_{1}\right)^{2}} .
$$

In unserem Beispiel aber sind:

$$
\sqrt{-\left(x_{4}-x_{1}\right)^{2}}=\frac{\sqrt{15}}{2} \quad \sqrt{-\left(x_{4}-x_{2}\right)^{2}}=\frac{\sqrt{3}}{2} \quad V-\left(x_{2}-x_{1}\right)^{2}=1
$$

und es gilt: $\sqrt{15}>2+\sqrt{3}$.

Nach diesen Vorbemerkungen können wir nun unser zweites Theorem formulieren und beweisen:

Theorem 2. Sei $A(x)$ ein hermitesches, skalares Feld, das die Wightman Axiome erfüllt mit evtl. Ausnahme des Lokalitätsaxioms, welches wir durch die folgende Voraussetzung ersetzen wollen:

$Z u$ jedem Punkt $(a)=\left(a_{12}, a_{13}, a_{14}, a_{24}, a_{34}\right) \in M=\left\{\left(a_{12}, a_{13}, a_{14}, a_{24}\right.\right.$, $\left.a_{34}\right) / a_{12}, a_{13}, a_{14}, a_{24}, a_{34}>0 ; 1, \sqrt{a_{12}}, \sqrt{a_{13}}, \sqrt{a_{14}}, \sqrt{a_{24}}, \sqrt{a_{34}}$ sind Kantenlängen 
eines Tetraeders

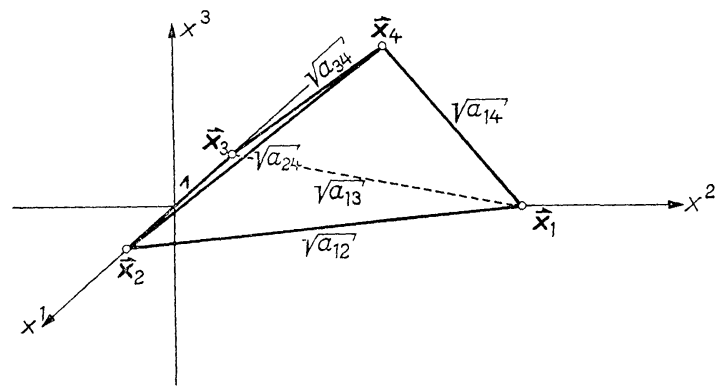

Fig. 2

das auch folgendermaßen entartet sein darf:

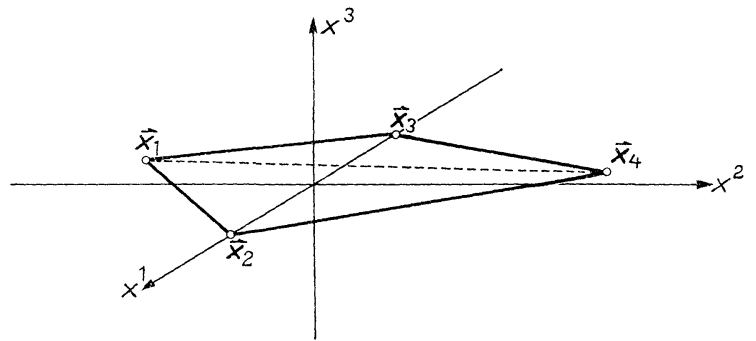

Fig. 3

gibt es Konstanten $C(a), h(a)>0$ so, da $\beta$

(V 2) $\left|\left\langle A\left(x_{1}\right)\left[A\left(x_{2}\right), A\left(x_{3}\right)\right] A\left(x_{4}\right)\right\rangle\right|<C(a) \exp \left\{-h(a) \sqrt{-z^{\alpha}}\right\}$ mit

$x_{1}=\left(\begin{array}{c}\frac{a_{13}-a_{12}}{2} \sqrt{-z} \\ \sqrt{a_{12}-\left[\frac{a_{12}-a_{13}+1}{2}\right]^{2}} \sqrt{-z} \\ 0\end{array}\right), x_{2}=\left(\begin{array}{c}0 \\ \frac{1}{2} \sqrt{-z} \\ 0 \\ 0\end{array}\right), x_{3}=\left(\begin{array}{c}0 \\ -\frac{1}{2} \sqrt{-z} \\ 0 \\ 0\end{array}\right.$

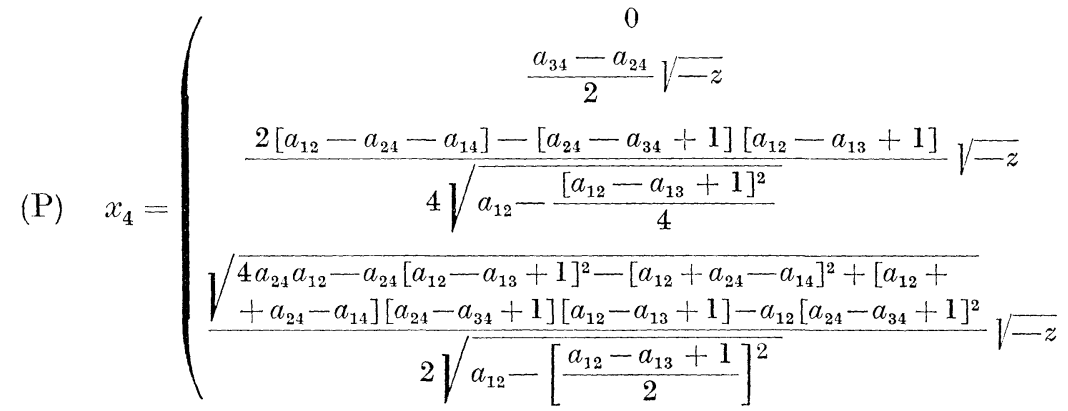

für reelles $-z>1$. 
Dann folgt für $\alpha>2$ :

$\left\langle A\left(x_{1}\right)\left[A\left(x_{2}\right), A\left(x_{3}\right)\right] A\left(x_{4}\right)\right\rangle=0 \quad$ für alle reellen Punkte $x_{1}, x_{2}, x_{3}, x_{4}$ mit $\left(x_{2}-x_{3}\right)^{2}<0$.

Der Beweis verläuft analog zu dem Beweis des ersten Theorems. Wir setzen:

$$
\begin{aligned}
& \zeta_{1}^{1}=-\frac{a_{12}-a_{13}+1}{2}, \quad \zeta_{1}^{2}=\sqrt{a_{12}-\frac{a_{12}-a_{13}+1}{2}}, \quad \zeta_{3}^{1}=\frac{a_{24}-a_{34}-1}{2}, \\
& \zeta_{3}^{2}=-\frac{\frac{a_{12}+a_{24}-a_{14}}{2}-\left[\frac{a_{24}-a_{34}+1}{2}\right]\left[\frac{a_{12}-a_{13}+1}{2}\right]}{\sqrt{a_{12}-\left[\frac{a_{12}-a_{13}+1}{2}\right]^{2}}},
\end{aligned}
$$$$
\zeta_{3}^{3}=-\frac{\sqrt{a_{12} a_{24}-a_{24}\left[\frac{a_{12}-a_{13}+1}{2}\right]^{2}-\left[\frac{a_{12}+a_{24}-a_{14}}{2}\right]^{2}+\left(a_{12}+a_{24}-\right.}}{\left.-a_{14}\right)\left[\frac{a_{24}-a_{34}+1}{2}\right]\left[\frac{a_{12}-a_{13}+1}{2}\right]-a_{12}\left[\frac{a_{24}-a_{34}+1}{2}\right]^{2}},
$$

$\zeta_{1}^{\prime 1}=\frac{a_{12}-a_{13}-1}{2}, \quad \zeta_{1}^{\prime 2}=-\zeta_{1}^{2}, \quad \zeta_{3}^{\prime 1}=-\frac{a_{24}-a_{34}+1}{2}$, $\zeta_{3}^{\prime 2}=-\zeta_{3}^{2}, \quad \zeta_{3}^{\prime 3}=-\zeta_{3}^{3}$.

Für $\Theta_{1}^{1,2,3,4}(a)<\Theta<2 \pi-\Theta_{1}^{1,2,3,4}(a)$ mit $\Theta_{1}^{1,2,3,4}(a)$

$=2 \operatorname{arctg}$

$$
\begin{aligned}
& {\left[\frac{\zeta_{3}^{1}}{\zeta_{3}^{3}}-\frac{\zeta_{3}^{2}}{\zeta_{3}^{3}}\left(\frac{\zeta_{1}^{1}}{\zeta_{1}^{2}}\right.\right.}\left.-\sqrt{\left.1+\left(\frac{\zeta_{1}^{1}}{\zeta_{1}^{2}}\right)^{2}\right)}+\sqrt{1+\left(\frac{\zeta_{3}^{1}}{\zeta_{3}^{3}}\right)^{2}+\left(\frac{\zeta_{3}^{2}}{\zeta_{3}^{3}}\right)^{2}}\right]^{2}+ \\
&+2 \sqrt{1+\left(\frac{\zeta_{1}^{1}}{\zeta_{1}^{2}}\right)^{2}}\left[\sqrt{1+\left(\frac{\zeta_{1}^{1}}{\zeta_{1}^{2}}\right)^{2}}-\frac{\zeta_{1}^{1}}{\zeta_{1}^{2}}\right]-1
\end{aligned}
$$

liegt $(\mathrm{P})$ in der "extended tube" zu der Reihenfolge 1, 2, 3, 4 bzw. für $\Theta_{1}^{1,3,2,4}(a)<\Theta<2 \pi-\Theta_{1}^{1,3,2,4}(a)$ mit

$\Theta_{1}^{1,3,2,4}(a)$

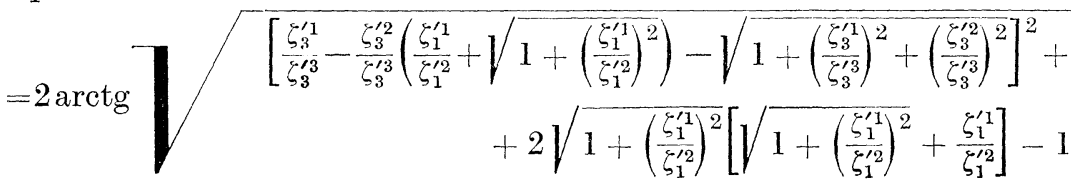

liegt (P) in der "extended tube" zu der Reihenfolge 1, 3, 2, 4. 
Die $S L(2, C)$-Matrizen $C_{1,2,3,4}$ bzw. $C_{1,3,2,4}$, die $(\mathrm{P})$ in die "primitive tubes" zu der Reihenfolge 1, 3, 2, 4 überführen, sind:

$$
\begin{aligned}
& C_{1,2,3,4}=\left(\begin{array}{l}
i \sigma\left\{-\frac{\zeta_{3}^{1}}{\zeta_{3}^{3}}+\frac{\zeta_{3}^{2}}{\zeta_{3}^{3}}\left(\frac{\zeta_{1}^{1}}{\zeta_{1}^{2}}-\sqrt{1+\left(\frac{\zeta_{1}^{1}}{\zeta_{1}^{2}}\right)^{2}}\right)-\sqrt{1+\left(\frac{\zeta_{3}^{1}}{\zeta_{3}^{3}}\right)^{2}+\left(\frac{\zeta_{3}^{2}}{\zeta_{3}^{3}}\right)^{2}},\right. \\
-\sigma\left(\frac{\zeta_{1}^{1}}{\zeta_{1}^{2}}-\sqrt{1+\left(\frac{\zeta_{1}^{1}}{\zeta_{1}^{2}}\right)^{2}}\right)-i \sigma,
\end{array}\right. \\
& \begin{array}{r}
\sigma\left(\frac{\zeta_{1}^{1}}{\zeta_{1}^{2}}-\sqrt{1+\left(\frac{\zeta_{1}^{1}}{\zeta_{1}^{2}}\right)^{2}}\right)-i \sigma \\
-i \sigma\left\{-\frac{\zeta_{3}^{1}}{\zeta_{3}^{3}}+\frac{\zeta_{3}^{2}}{\zeta_{3}^{3}}\left(\frac{\zeta_{1}^{1}}{\zeta_{1}^{2}}-\sqrt{1+\left(\frac{\zeta_{1}^{1}}{\zeta_{1}^{2}}\right)^{2}}\right)-\sqrt{1+\left(\frac{\zeta_{3}^{1}}{\zeta_{3}^{3}}\right)^{2}+\left(\frac{\zeta_{3}^{2}}{\zeta_{3}^{3}}\right)^{2}}\right\}
\end{array} \\
& C_{1,3,2,4}=\left\{\begin{array}{l}
i \tau\left\{-\frac{\zeta_{3}^{\prime 1}}{\zeta_{3}^{\prime 3}}+\frac{\zeta_{3}^{\prime 2}}{\zeta_{3}^{\prime \prime}}\left(\frac{\zeta_{1}^{\prime 1}}{\zeta_{1}^{\prime 2}}+\sqrt{1+\left(\frac{\zeta_{1}^{\prime 1}}{\zeta_{1}^{\prime 2}}\right)^{2}}\right)+\sqrt{1+\left(\frac{\zeta_{3}^{\prime 1}}{\zeta_{3}^{\prime \prime}}\right)^{2}+\left(\frac{\zeta_{3}^{\prime 2}}{\zeta_{3}^{\prime 3}}\right)^{2}},\right. \\
-\tau\left(\frac{\zeta_{1}^{\prime 1}}{\zeta_{1}^{\prime 2}}+\sqrt{1+\left(\frac{\zeta_{1}^{\prime 1}}{\zeta_{1}^{\prime 2}}\right)^{2}}\right)-i \tau,
\end{array}\right.
\end{aligned}
$$

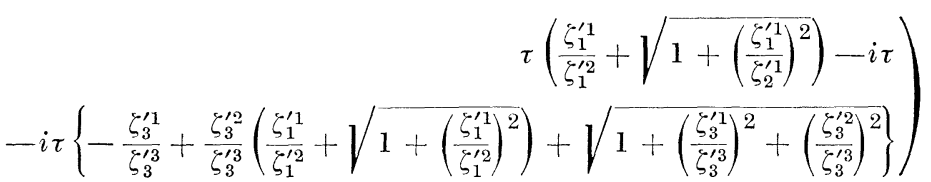

$$
\sigma^{-1}=\sqrt{\left[\begin{array}{r}
{\left[\frac{\zeta_{3}^{1}}{\zeta_{3}^{3}}-\frac{\zeta_{3}^{2}}{\zeta_{3}^{3}}\left(\frac{\zeta_{1}^{1}}{\zeta_{1}^{2}}-\sqrt{\left.\left.1+\left(\frac{\zeta_{1}^{1}}{\zeta_{1}^{2}}\right)^{2}\right)+\sqrt{1+\left(\frac{\zeta_{3}^{1}}{\zeta_{3}^{3}}\right)^{2}+\left(\frac{\zeta_{3}^{2}}{\zeta_{3}^{3}}\right)^{2}}\right]^{2}+}\right.\right.} \\
+2 \sqrt{1+\left(\frac{\zeta_{1}^{2}}{\zeta_{1}^{1}}\right)^{2}}\left[\sqrt{1+\left(\frac{\zeta_{1}^{1}}{\zeta_{1}^{2}}\right)^{2}}-\frac{\zeta_{1}^{1}}{\zeta_{1}^{2}}\right]
\end{array}\right.}
$$

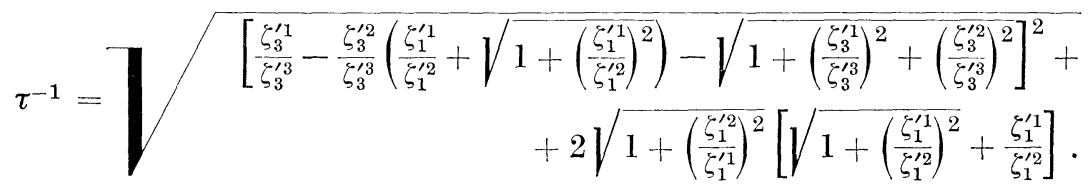

Der Punkt (P) liegt also im Durchschnitt der "extended tubes" zu den beiden Reihenfolgen für $\Theta_{1}(a)<\Theta<\Theta_{3}(a)=2 \pi-\Theta_{1}(a)$

$$
\Theta_{1}(a)=\operatorname{Max}\left\{\Theta^{1,2,3,4}(a), \Theta^{1,2,3,4}(a)\right\} .
$$

Zu jedem $\alpha>2$ gibt es nun eine offene Menge $O \subset M$, so daß $\Theta_{1}(a)<\pi-\frac{\pi}{\alpha}$ für alle $(a) \in O$ (Konfigurationen in der Nähe von der symmetrischen, 
entarteten Sitation

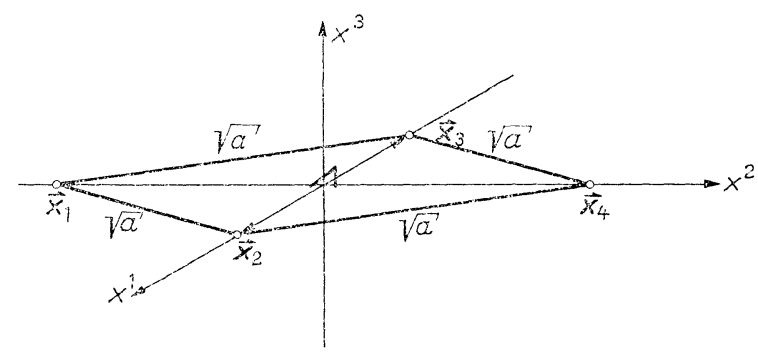

Fig. 4

für hinreichend großes a).

Daraus können wir wieder schließen (vgl. S. 7) ff

a) $\left\langle A\left(x_{1}\right)\left[A\left(x_{2}\right), A\left(x_{3}\right)\right] A\left(x_{4}\right)\right\rangle=0$ für alle reellen $x_{1}, x_{2}, x_{3}, x_{4}$ mit $\left(x_{2}-x_{3}\right)^{2}<0$.

Bei den Behandlungen von $\left\langle\left[A\left(x_{1}\right), A\left(x_{2}\right)\right] A\left(x_{3}\right) A\left(x_{4}\right)\right\rangle$ und $\left\langle A\left(x_{1}\right) A\left(x_{2}\right)\left[A\left(x_{3}\right), A\left(x_{4}\right)\right]\right\rangle$ - wir machen die (V 2) entsprechenden Voraussetzungen - gehen wir analog vor und folgern:

b) $\left\langle\left[A\left(x_{1}\right), A\left(x_{\ddot{z}}\right)\right] A\left(x_{3}\right) A\left(x_{4}\right)\right\rangle=0 \quad$ für alle reellen $x_{1}, x_{2}, x_{3}, x_{4}$ und mit $\left(x_{1}-x_{2}\right)^{2}<0$

c) $\left\langle A\left(x_{1}\right) A\left(x_{2}\right)\left[A\left(x_{3}\right), A\left(x_{4}\right)\right]\right\rangle=0$

für alle reellen $x_{1}, x_{2}, x_{3}, x_{4}$ mit $\left(x_{3}-x_{4}\right)^{2}<0$.

a), b) und c) bedeuten aber gerade strikte Lokalität für die Vierpunktfunktion.

Felder mit Akausalitäten von der Art (V 2) führen auf dieselben Eigenschaften für die Vierpunktfunktion wie strikt lokale Felder.

\section{Allgemeine $n$-Punktiunktion}

Es ist nun ziemlich klar, wie wir das Beweisverfahren auf die $n$-Punktfunktion für allgemeines $n$ übertragen können.

Theorem 3. Sei $A(x)$ ein hermitesches, skalares Feld, das die Wightman Axiome erfüllt mit evtl. Ausnahme des Lokalitätsaxioms, welches wir durch die folgende Voraussetzung ersetzen wollen:

$Z$ Zujeder reellen Konfiguration $(X): X_{1}, \ldots, X_{j-1}, X_{j}, X_{j+1}, X_{j+2}, \ldots, X_{n}$, die in den Reihenfolgen $1, \ldots, j-1, j, j+1, j+2, \ldots, n$ und $1, \ldots, j-1$, $j+1, j, j+2, \ldots, n$ total raumartig ist, gibt es Konstanten $0<C(X), h(X)$ so, daß für $x_{k}=X_{k} \cdot V-z$ und reelles $-z>1$ gilt:

(V) $\left|\left\langle A\left(x_{1}\right) \ldots A\left(x_{j-1}\right)\left[A\left(x_{j}\right), A\left(x_{j+1}\right)\right] A\left(x_{j+2}\right) \ldots A\left(x_{n}\right)\right\rangle\right|<$

$$
<C(X) \exp \left\{-h(X) /-z^{\alpha}\right\} \text {. }
$$


Dann folgt für $\alpha>2:\left\langle A\left(x_{1}\right) \ldots A\left(x_{j-1}\right)\left[A\left(x_{j}\right), A\left(x_{j+1}\right)\right] A\left(x_{j+2}\right) \ldots\right.$ $\left.\ldots A\left(x_{n}\right)\right\rangle=0$ für alle reellen Punkte $x_{1}, \ldots, x_{j-1}, x_{j}, x_{j+1}, x_{j+2}, \ldots, x_{n}$ mit $\left(x_{j}-x_{j+1}\right)^{2}<0$.

Zum Beweis wähle man $(X)$ in der Nähe von der folgenden Konfiguration:

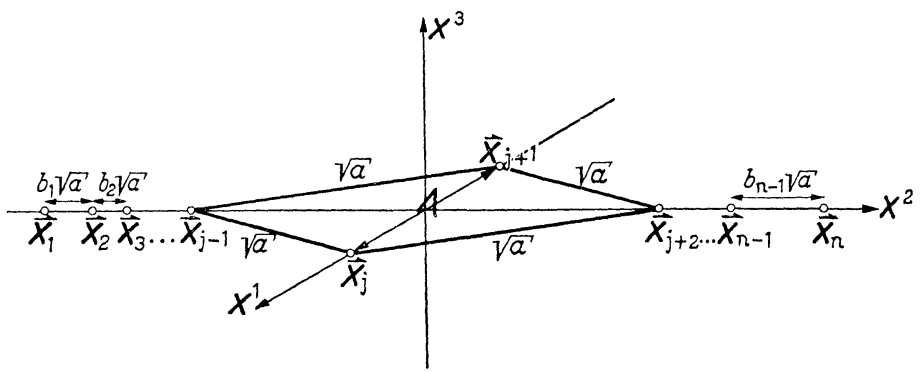

Fig. 5

wobei besonders der Fall $a \gg 1$ interessiert.

Herrn Prof. H. J. Borchers sei für eine klärende Diskussion und Herrn B. Humpert für seine Hilfe bei den Rechnungen gedankt.

\section{Literatur}

1. Streater, R. F., and A. S. Wightman: PCT, spin statistics, and all that. p. 134.

New York-Amsterdam: W. A. Benjamin, Inc. 1964.

2. Jost, R.: Eine Bemerkung zum CTP Theorem. Helv. Phys. Acta 30, 409 (1957).

3. Källén, G., and A. S. Wightman: Dan. Vid. Selsk. Mat. Fys. Skr. 1, nº 6 (1958).

4. BoAs, R. P.: Entire functions, chap. 5, sec. 1, theorem 12. New York: Academic

Press Inc. 1954. 